\title{
Salvage Radiotherapy for Solitary Metachronous Bone Metastasis in Patients with Breast Cancer
}

\author{
MARI MIYATA $^{1}$, TAKAYUKI OHGURI ${ }^{1}$, KATSUYA YAHARA $^{1}$, \\ SHINSAKU YAMAGUCHI ${ }^{2}$, HAJIME IMADA ${ }^{3}$ and YUKUNORI KOROGI ${ }^{1}$ \\ ${ }^{1}$ Department of Radiology, University of Occupational and Environmental Health, Kitakyushu, Japan; \\ ${ }^{2}$ Department of Radiology, Kitakyushu General Hospital, Kitakyushu, Japan; \\ ${ }^{3}$ Department of Cancer Therapy Center, Tobata Kyoritsu Hospital, Kitakyushu, Japan
}

\begin{abstract}
Aim: We assessed the efficacy and toxicity of salvage radiotherapy for solitary metachronous bone metastasis (SMBM) in patients with breast cancer. Patients and Methods: We retrospectively analyzed 17 patients with $S M B M$ who were treated with salvage radiotherapy. First failure was detected as SMBM in all patients. Salvage radiotherapy using three-dimensional treatment planning was performed at a median total dose of 50 Gy. Median daily dose was $2.0 \mathrm{~Gy}$. Results: Median follow-up was 40 months. Local recurrence of SMBM was noted in only two patients. The 3-year overall survival, progression-free survival, and local control rates were $93 \%, 51 \%$, and $85 \%$, respectively. Median overall and progression-free survival were 74 and 30 months, respectively. Toxicities were mild, and bone fractures were not observed. Conclusion: Salvage radiotherapy for SMBM was able to achieve higher local control rates without severe toxicity, as well as to provide longer progression-free survival; therefore, this may be an effective modality.
\end{abstract}

Skeletal involvement is present in more than half of breast cancer cases with distant metastasis (1). The development of methods for early detection of recurrence or distant metastases, such as computed tomography (CT), magnetic resonance imaging (MRI), positron-emission tomography (PET), and bone scintigraphy, allows for detection of limited bone metastasis. Hormonal therapy, chemotherapy, external radiotherapy (RT), and bisphosphonate therapy are the main therapeutic options for bone metastases from breast cancer.

Correspondence to: Dr. Takayuki Ohguri, Department of Radiology, University of Occupational and Environmental Health, 1-1 Iseigaoka, Yahatanishi-ku, Kitakyushu 807-8555, Japan. Tel: +81 936917264, Fax: +81 936920249, e-mail: ogurieye@med.uoeh-u.ac.jp

Key Words: Breast cancer, bone metastasis, radiotherapy, oligometastases.
Hellmann et al. defined the term 'oligometastases' as a limited metastasis with a maximum of 3-4 clinically detectable metastases (2). Patients with oligometastases have a better prognosis, and local therapy, such as salvage RT and surgery, has an important role in further development of the disease (3-9). Distant metastasis in patients with breast cancer tends to occur in the lung, bone, liver, brain, and lymph nodes (10). Milano et al. reported 85 metastatic lesions in 40 breast cancer patients treated with stereotactic body radiotherapy (SBRT) and that the most favorable prognostic factor for patients with oligometastatic breast cancer was metastases involving only the bone (11). Furthermore, metachronous metastases in patients with oligometastatic disease were significant prognostic factors for better overall survival (OS) rates compared to patients with synchronous metastases (12). These findings indicated that salvage RT for metachronous oligometastases of the bone could contribute to clinical outcomes in patients with breast cancer. Therefore, in many cases, salvage RT has been selected to treat solitary metachronous bone metastases (SMBM) at our Institutions. However, there are few reports of salvage RT in patients with breast cancer with SMBM $(11,13)$. The purpose of this study was to assess the efficacy and toxicity of salvage RT for SMBM in patients with breast cancer.

\section{Patients and Methods}

Patients. From April 2002 to March 2014, 274 patients with metastatic breast cancer were recorded prospectively in the database for RT at our institutions. All 17 consecutive patients with breast cancer with SMBM who were treated with salvage RT satisfied the following requirements of our definition of SMBM and were included in this retrospective study: (i) pathologically confirmed breast carcinoma; (ii) disease-free status after initial therapy for clinically localized breast cancer had been confirmed once based on the results of a physical examination, postoperative pathological findings, and CT; and (iii) first failure was detected as SMBM. Written informed consent for treatment was obtained from all patients. The study was approved by Institutional Review Boards of our institutions. 
Table I. Patient characteristics ( $n=17)$.

\begin{tabular}{lc}
\hline Variable & Value \\
\hline Median age (range), years & $70(40-87)$ \\
Period between the initial surgery & \\
and SMBM (range), months & $65(3-252)$ \\
Initial treatment for primary breast cancer, n (\%) & \\
$\quad$ Total resection & $9(53)$ \\
Partial resction & $8(47)$ \\
Bone lesion, n (\%) & $9(53)$ \\
Lytic & $5(29)$ \\
Blastic & $3(18)$ \\
Mixed (lytic/blastic) & \\
Site of SMBM, n (\%) & $5(29)$ \\
Spine & $5(29)$ \\
Pelvic bone & $4(24)$ \\
Sternum & $1(6)$ \\
Rib & $1(6)$ \\
Humerus & $1(6)$ \\
Patella &
\end{tabular}

SMBM: Solitary metachronous bone metastasis.

The patient characteristics for SMBM are listed in Table I. Four out of the 17 patients were diagnosed pathologically as having SMBM by bone biopsy. In the other patients, diagnosis was based on longitudinal $\mathrm{CT}$ and tumor marker expression. In some cases, ${ }^{18} \mathrm{~F}$-fluorodeoxyglucose-PET/CT or MRI were also used.

The tumor/node/metastasis (TNM) stages (based on the International Union Against Cancer TNM classification, sixth edition) were evaluated pathologically at the initial surgery: Stage IIA was noted in three patients, stage IIB in five, IIIA in three, and IIIB in three, while the stage was unknown in three. After the initial surgery, eight patients were treated with postoperative RT, while eight received adjuvant chemotherapy as follows: cyclophosphamide plus adriamycin in three patients; cyclophosphamide, epirubicin, and 5-fluorouracil (FEC) in two; trastuzumab in two; and cyclophosphamide, methotrexate, 5-fluorouracil, and doxifluridine in one (14). Six patients were treated with hormonal therapy after the initial surgery. Three patients were treated with neoadjuvant chemotherapy, including FEC and docetaxel, before the initial surgery.

Treatments methods for SMBM. The treatment methods for SMBM are summarized in Table II. All 17 patients were treated with external RT. The total radiation dose of the salvage RT, using a 4, 6 , or $10 \mathrm{MV}$ linear accelerator, ranged from 40 to $74 \mathrm{~Gy}$ (median=50 Gy), and the daily dose was 1.8 to $2.0 \mathrm{~Gy}$ (median=2.0 Gy). CT-assisted three-dimensional treatment planning (Xio or FOCUS; CMS Japan, Tokyo, Japan) was used to determine the RT fields in all 17 patients; the clinical target volume (CTV) of spinal metastases encompassed the gross target volume (GTV) on CT, MRI, or PET-CT and the surrounding vertebral body, including superior and inferior endplates and any existing paraspinal component plus 0.5 to $1.0 \mathrm{~cm}$. The GTV of other bone metastases was defined as the gross visible lesion on diagnostic CT, MRI, or PET-CT, and CTV encompassed the GTV plus 0.5 to $1.0 \mathrm{~cm}$ of contiguous bone and soft tissue extension if present. The planning target volume (PTV) was the CTV plus 0.5 to $1.5 \mathrm{~cm}$ for the daily
Table II. Treatment methods for solitary metachronous bone metastasis $(S M B M)$.

\begin{tabular}{lc}
\hline Variable & Value \\
\hline Salvage RT & $17(100)$ \\
Median total dose (range), Gy & $50(40-74)$ \\
Median daily dose (range), Gy & $2.0(1.8-2.0)$ \\
Decompressive surgery and spine & \\
stabilization before salvage RT, $\mathrm{n}(\%)$ & $1(6)$ \\
Systemic therapy for SMBM before & \\
salvage RT, n (\%) & $4(24)$ \\
Hormonal therapy, $\mathrm{n}$ & 2 \\
Chemotherapy, $\mathrm{n}$ & \\
$\quad$ Docetaxel & 1 \\
CEF & 1 \\
Response to systemic therapy immediately \\
before salvage RT, $\mathrm{n}$ \\
$\quad$ SD \\
PD \\
Concurrent systemic therapy during \\
salvage RT, $\mathrm{n}(\%)$ \\
Hormonal therapy, $\mathrm{n}$ \\
Chemotherapy, docetaxel, $\mathrm{n}$ \\
Adjuvant systemic therapy after \\
salvage RT, n (\%) \\
Hormonal therapy, $\mathrm{n}$ \\
Chemotherapy, paclitaxel, n & 1 \\
\hline
\end{tabular}

RT: Radiotherapy; CEF: cyclophosphamide, epirubicin and fluorouracil; SD: stable disease; PD: progressive disease.

setup variation and respiratory movement. The field area of the CTassisted three-dimensional treatment planning covered the PTV with a two-, three-, or four-field, or conformational technique.

Systemic therapy for SMBM before, during, and after the salvage RT is summarized in Table II. In addition, zoledronic acid was administered to two patients, and denosumab to one.

Evaluation and follow-up. Follow-up evaluations were performed by CT every 1 to 6 months. The OS, progression-free survival (PFS), and local control (LC; defined as failure to have a recurrence within the RT field for SMBM) rates were calculated from the start of the salvage RT for SMBM using the Kaplan-Meier method. The National Cancer Institute Common Toxicity Criteria version 4 (CTCAE) was used to score the patient toxicity (15). The highest toxicity grade obtained for each patient was used for the toxicity analysis. The toxicity was defined as acute (during therapy and up to 3 months after salvage RT) or late (over 3 months after the completion of salvage RT).

\section{Results}

The median follow-up after salvage RT for SMBM was 40 months (range $=8-160$ months). All patients completed the planned RT. The first site of disease progression after salvage RT for SMBM is summarized in Table III. The first site of disease progression was out-of-field in 10 patients $(59 \%$; multiple bones in four patients, thoracic vertebra in one, tibia 
Table III. First site of disease progression after salvage radiotherapy for solitary metachronous bone metastasis.

\begin{tabular}{lc}
\hline Site & $\mathrm{n}(\%)$ \\
\hline Out-of-field & $10(59)$ \\
Bone, $\mathrm{n}$ & 6 \\
Liver, $\mathrm{n}$ & 2 \\
Other, $\mathrm{n}$ & 2 \\
Out-of-field + within radiation field & $2(12)$ \\
No disease progression & $5(29)$ \\
\hline
\end{tabular}

in one, liver in two, and multiple organs in two), and was both within radiation field and out-of-field in two patients (12\%; out-of-field of disease progression was seen in multiple bones in one patient and multiple organs in one). There was no patient in whom the first site of disease progression was local (in-field) alone. During the follow-up period, disease progression was not recognized in five patients $(29 \%)$.

The 3-year OS, PFS, and LC (in-field) rates were 93\%, $51 \%$, and $85 \%$, respectively (Figure 1). The 5-year OS, PFS, and LC (in-field) rates were $73 \%, 33 \%$, and $85 \%$, respectively. The median survival times with regard to OS and PFS rates after salvage RT for the SMBM were 74 and 30 months, respectively. All nine patients with pain, considered to be related to SMBM, had symptom relief (vanished in four, diminished in five).

The observed toxicities were mild. Acute toxicities grade 2 or more occurred in three patients (18\%); grade 3 dermatitis in one, grade 2 dermatitis in one, and grade 2 esophagitis in one. No late toxicities of grade 2 or more, including bone fracture and bone necrosis, were observed.

A representative case is shown in Figure 2.

\section{Discussion}

As most cases of metastatic breast cancer are not curable, the standard management of these patients has been systemic chemotherapy (16). However, as mentioned previously, local therapy may have an important role in patients with SMBM. Previous studies of salvage RT in patients with breast cancer with SMBM were very limited by a small number of treated patients $(11,13)$. To our knowledge, the present study is the largest series evaluating salvage RT for patients with SMBM from breast cancer. Milano et al. conducted a prospective pilot study to assess patient outcome after SBRT for oligometastases from breast cancer. In their prospective study, eight patients had bone-only metastases; seven out of the eight had no evidence of disease recurrence, and patients with the bone metastases experienced a significantly improved PFS compared to those with other sites of

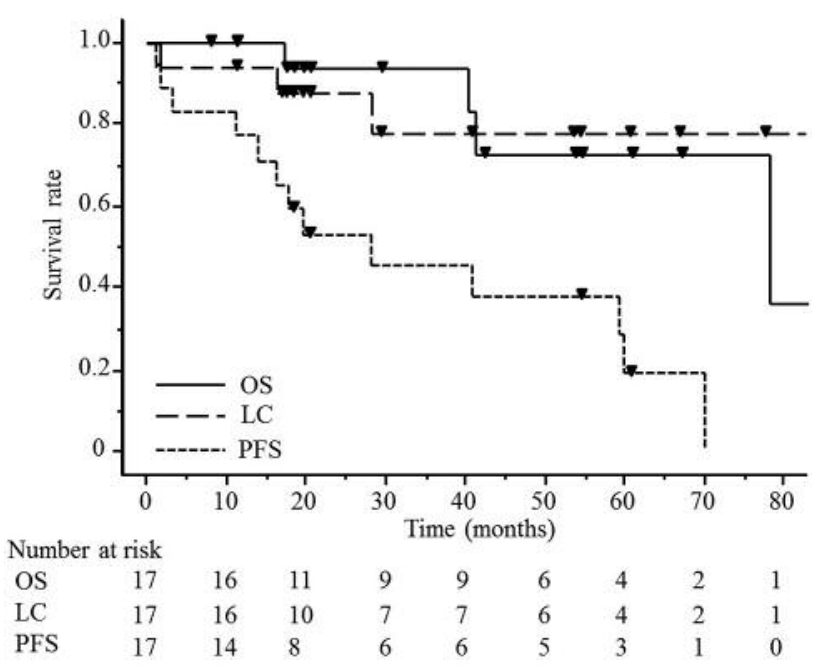

Figure 1. Rates of overall survival (OS), local (in-field) control (LC), and progression-free survival (PFS) after salvage radiotherapy for solitary metachronous bone metastasis from breast cancer.

oligometastases (11). Niibe et al. reported on solitary bone metastases in seven patients treated with conventional highdose RT. All seven patients were alive at the last follow-up; only one had pain relapse, and the median total RT dose was 46 Gy [30-50 Gy; biological effective dose (BED), 39-60 Gy10] (13). In the present study, salvage RT, using conventional high-dose RT with a median total RT dose of 50 Gy (BED=60 Gy10) for patients with SMBM, also resulted in high LC rates of $85 \%$. Long-term PFS time of 30 months were promising without severe toxicity.

Recently, SBRT for spinal bone metastasis has been applied more frequently; however, vertebral compression fracture (VCF) has become increasingly recognized as an adverse event after SBRT $(17,18)$. Sahgal et al. summarized the current evidence of VCF after spinal SBRT, and crude risk estimates for VCF after spinal SBRT range from $11 \%$ to $39 \%$ (18), although VCF is a fairly lowrisk adverse event (approximately 5\% risk) after conventional RT. Cunha et al. analyzed the risk of VCF after spine SBRT and concluded that the presence of kyphotic/scoliotic deformity and the presence of lytic tumor were the only factors predictive of $\mathrm{VCF}$, and that treatment with SBRT of $20 \mathrm{~Gy}$ or greater in a single fraction conferred a higher risk of VCF (17). In the present study, VCF or fractures of other bones after salvage RT were not recognized in any patient. We suppos that salvage RT using high-dose with conventional fractions in the present study was promising in patients with SMBM, especially for those with the aforementioned risk factors for fracture, because long-term survival times and high LC rate were achieved without bone fracture. 

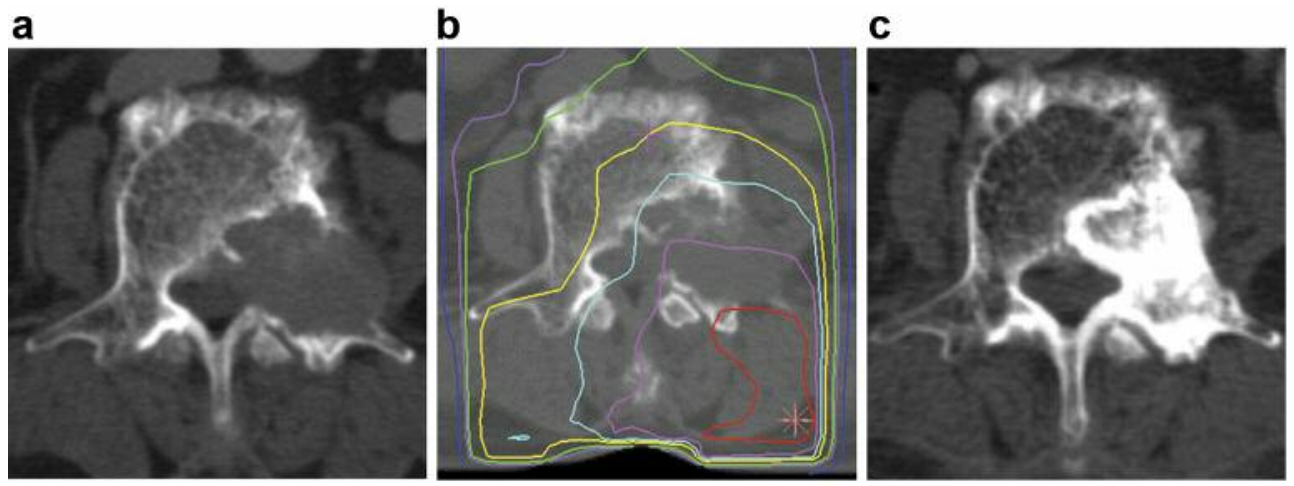

Figure 2. A 62-year-old woman was treated with breast-conserving therapy for stage pT2NOMO breast cancer. Although there was no evidence of disease 102 months after the breast conservation therapy, solitary metachronous bone metastasis of the third lumbar spine from breast cancer was diagnosed using bone biopsy (a). Salvage radiotherapy of $60 \mathrm{~Gy}$ in 30 fractions was performed for the lumbar bone metastasis. Computed tomography with dose distribution is shown: Red, magenta, light blue, yellow, green, purple, and blue lines denote 65, 60, 55, 50, 40, 35 and $20 \mathrm{~Gy}$, respectively (b). Osteo-anagenesis was seen on computed tomography 48 months after the salvage radiotherapy. There was no evidence of disease at the 55-month follow-up after salvage radiotherapy $(c)$.

In the present study, long-term PFS and high LC rates were recognized after salvage RT for SMBM without severe toxicities. Nevertheless, we believe that an additional systemic treatment may also be necessary for SMBM, because the first sites of disease progression were recognized as being out-of field of salvage RT for SMBM in all 12 patients who experienced disease progression. Recently, patterns of recurrence after curative-intent RT for oligometastases confined to one organ indicated that new metastases occurred in $73 \%$ of patients (19). Previously reported favorable prognostic patients with factors of recurrent/metastatic breast cancer were as follows: younger age, complete remission after initial therapy for breast cancer, soft-tissue metastasis, smaller recurrent/metastatic tumor volume, and a longer period between the initial surgery and recurrence/metastasis (20). Therefore, we speculate that additional systemic treatment should be selected, particularly for SMBM without the aforementioned prognostic factors.

Certain limitations are associated with this study. Because it was a small retrospective case series with heterogeneous treatment, the possibility of selection bias cannot be ruled out. A formal prospective trial is needed to determine the efficacy and prognostic factors of this therapy in patients with breast cancer with SMBM.

In conclusion, salvage RT in patients with breast cancer with SMBM achieved a high LC rate without severe toxicity, and provided long-term PFS. Therefore, salvage RT is a promising treatment in patients with SMBM from breast cancer. Further prospective evaluations with detailed treatment protocols are necessary to clarify whether salvage RT could improve clinical outcomes in patients with breast cancer with SMBM.

\section{References}

1 Coleman RE: Clinical features of metastatic bone disease and risk of skeletal morbidity. Clin Cancer Res 12: 6243s-6249s, 2006.

2 Hellman S and Weichselbaum RR: Oligometastases. J Clin Oncol 13: 8-10, 1995.

3 Rades D, Dziggel L, Janssen S, Blanck O, Hornung D and Schild SE: A Survival score for patients receiving stereotactic radiosurgery alone for brain metastases from breast cancer. Anticancer Res 36: 1073-1076, 2016.

4 Janssen S and Rades D: Primary breast cancer with synchronous metastatic disease - indications for local radiotherapy to the breast and chest wall. Anticancer Res 35: 5807-5812, 2015.

5 Pasqualetti F, Montrone S, Vivaldi C, Zani M, Fedele D, Fornaro L, Pasqualetti G, Salvatore L, Manfredi B, Laliscia C, Coraggio G, Gonnelli A, Loupakis F, Masi G, Sainato A, Monzani F, Falcone A and Paiar F: Stereotactic body radiotherapy in patients with lung oligometastases from colorectal cancer. Anticancer Res 37: 315-319, 2017.

6 Niibe Y, Yamashita H, Sekiguchi K, Takahashi W, Shiraishi K, Okuma K, Terahara A, Kawamori J and Nakagawa K: Stereotactic body radiotherapy results for pulmonary oligometastases: a twoinstitution collaborative investigation. Anticancer Res 35: 49034908, 2015.

7 Palma DA, Louie AV and Rodrigues GB: New strategies in stereotactic radiotherapy for oligometastases. Clin Cancer Res 21: 5198-5204, 2015.

8 Planchard D, Soria JC, Michiels S, Grunenwald D, Validire P, Caliandro R, Girard P and Le Chevalier T: Uncertain benefit from surgery in patients with lung metastases from breast carcinoma. Cancer 100: 28-35, 2004.

9 McDonald ML, Deschamps C, Ilstrup DM, Allen MS, Trastek VF and Pairolero PC: Pulmonary resection for metastatic breast cancer. Ann Thorac Surg 58: 1599-1602, 1994.

10 Singletary SE, Walsh G, Vauthey JN, Curley S, Sawaya R, Weber KL, Meric F and Hortobagyi GN: A role for curative 
surgery in the treatment of selected patients with metastatic breast cancer. Oncologist 8: 241-251, 2003.

11 Milano MT, Zhang H, Metcalfe SK, Muhs AG and Okunieff P: Oligometastatic breast cancer treated with curative-intent stereotactic body radiation therapy. Breast Cancer Res Treat 115 : 601-608, 2009.

12 Ashworth AB, Senan S, Palma DA, Riquet M, Ahn YC, Ricardi U, Congedo MT, Gomez DR, Wright GM, Melloni G, Milano MT, Sole CV, De Pas TM, Carter DL, Warner AJ and Rodrigues GB: An individual patient data metaanalysis of outcomes and prognostic factors after treatment of oligometastatic non-smallcell lung cancer. Clin Lung Cancer 15: 346-355, 2014.

13 Niibe Y, Kuranami M, Matsunaga K, Takaya M, Kakita S, Hara T, Sekiguchi K, Watanabe M and Hayakawa K: Value of high-dose radiation therapy for isolated osseous metastasis in breast cancer in terms of oligo-recurrence. Anticancer Res 28: 3929-3931, 2008.

14 Greene F, Page D, Fleming I, Fritz A, Balch C and Haller D: American Joint Committee on Cancer. AJCC Cancer Staging Manual. 6th ed. New York: Springer-Verlag, 2002.

15 National Cancer Institute. National Cancer Institute Common Terminology Criteria for Adverse Events (CTCAE) v.4.0. 2010.

16 Tiainen L, Tanner M, Lahdenpera O, Vihinen P, Jukkola A, Karihtala P, Paunu N, Huttunen T and Kellokumpu-Lehtinen PL: Bevacizumab combined with docetaxel or paclitaxel as first-line treatment of HER2-negative metastatic breast cancer. Anticancer Res 36: 6431-6438, 2016.
17 Cunha MV, Al-Omair A, Atenafu EG, Masucci GL, Letourneau D, Korol R, Yu E, Howard P, Lochray F, da Costa LB, Fehlings MG and Sahgal A: Vertebral compression fracture (VCF) after spine stereotactic body radiation therapy (SBRT): analysis of predictive factors. Int J Radiat Oncol Biol Phys 84: e343-349, 2012.

18 Sahgal A, Whyne CM, Ma L, Larson DA and Fehlings MG: Vertebral compression fracture after stereotactic body radiotherapy for spinal metastases. Lancet Oncol 14: e310-320, 2013.

19 Milano MT, Katz AW and Okunieff P: Patterns of recurrence after curative-intent radiation for oligometastases confined to one organ. Am J Clin Oncol 33: 157-163, 2010.

20 Hortobagyi GN: Can we cure limited metastatic breast cancer? J Clin Oncol 20: 620-623, 2002.
Received March 2, 2017

Revised March 19, 2017

Accepted March 21, 2017 